\title{
Novos biótipos pentaplóides do grupo Dilatata de Paspalum L. (Gramineae) no Sul do Brasil ${ }^{1}$
}

\author{
New pentaploid biotypes of the Dilatata group of Paspalum L. (Gramineae) from \\ Southern Brazil
}

\author{
Ana Caroline de Carvalho Machado ${ }^{2}$ José Francisco Montenegro Valls $^{3}$ \\ Andréa del Pilar de Souza Peñaloza ${ }^{4}$ Sileuza dos Santos ${ }^{5}$
}

\section{RESUMO}

Paspalum dilatatum é uma gramínea nativa da América do Sul subtropical, com bom valor forrageiro e ampla variabilidade intraespecífica. Distintos tipos morfológicos, citológicos e reprodutivos têm sido citados para esta espécie. Paspalum dilatatum "Comum" é o biótipo de maior interesse agronômico. A constatação da importância da espécie como componente da produção de forragem de boa qualidade em campos naturais vem resultando em contínuo interesse pela possibilidade de seu melhor aproveitamento em cultivo. Entre o material do grupo Dilatata submetido à análise, seis acessos de classificação taxonômica não definida apresentaram morfologia distinta dos demais. Acredita-se que sejam resultantes de processos de hibridação natural em combinações antes desconhecidas. A análise mitótica evidenciou que todos possuem $2 n=50$ cromossomos. Três destes acessos mostraram características morfológicas intermediárias entre os biótipos "Virasoro" (4x, sexual) e "Uruguaiana" (6x, apomítico). A confirmação de ocorrência geográfica próxima reforça a hipótese de hibridação e evidencia uma ampliação da área de ocorrência do biótipo sexual envolvido neste cruzamento. Os outros acessos são, provavelmente, híbridos naturais entre P. dilatatum "Torres" e P. urvillei. Tais acessos mostram a inflorescência com eixo mais longo e com mais ramos que as plantas típicas do biótipo "Torres". A caracterização morfológica e citológica do material discrepante poderá levar ao estabelecimento de parâmetros seguros para sua diferenciação, que permitam sua inclusão em categorias taxonômicas adequadas. Sugere-se a origem destes novos biótipos pentaplóides distintos do "Comum", a partir de cruzamentos ocorridos no Sul do Brasil entre outros dois biótipos e espécies do grupo.
Palavras-chave: Paspalum dilatatum, biótipo, pentaplóide, hibridação.

\begin{abstract}
Paspalum dilatatum is a native grass in subtropical South America, with good forage value and broad intraspecific variability. Distinct morphological, cytological and reproductive types have been cited for the species. "Common" $\boldsymbol{P}$. dilatatum is the biotype of greatest agronomic interest. The assessment of the species importance as a component of good quality forage production in natural pastures has resulted in continuous interest for the possibility of its enhanced use in cultivation. Among materials of the Dilatata group analyzed here, six accessions of uncertain taxonomic classification showed a distinct morphology. We believe they result from natural hybridization processes in yet unknown combinations. Mitotic analysis showed they all have $2 n=50$ chromosomes. Three of these accessions showed intermediate morphological features between "Virasoro" (4x, sexual) and "Uruguaiana" (6x, apomitic) biotypes. Confirmation of close geographic occurrence stresses the hybridization hypothesis, and broadens the known area of occurrence of the sexual biotype involved in this cross. The other accessions are, most probably, natural hybrids between the "Torres" biotype of $\boldsymbol{P}$. dilatatum and $\boldsymbol{P}$. urvillei. Such accessions have inflorescences with a larger axis and more branches than typical plants of "Torres" biotype. Morphological and cytological characterization of this different materials may lead to the establishment of reliable parameters for their differentiation and possible assignment to an adequate taxonomic category. We suggest that these
\end{abstract}

${ }^{1}$ Parte da dissertação de Mestrado em Botânica da primeira autora, Universidade de Brasília (UnB), Brasília, DF

${ }^{2}$ Biólogo, aluno de Mestrado em Botânica, UnB, Bolsista Coordenação de Aperfeiçoamento de Pessoal de Nível Superior (CAPES). ${ }^{3}$ Engenheiro Agrônomo, PhD, Embrapa Recursos Genéticos e Biotecnologia, Parque Estação Biológica, Final W5 Norte, CP 02372, 70770-900, Brasília, DF, Brasil, Bolsista de Produtividade em Pesquisa do Conselho Nacional de Desenvolvimento Científico e Tecnológico (CNPq). E-mail: valls@ cenargen.embrapa.br. Autor para correspondência.

${ }^{4}$ Engenheiro Agrônomo, Doutor, Embrapa Recursos Genéticos e Biotecnologia.

${ }^{5}$ Assistente de Operações, Embrapa Recursos Genéticos e Biotecnologia. 
new pentaploid biotypes, distinct from the "Common" biotype, derived from crosses that occurred in Southern Brazil between other biotypes and species of the group.

Key words: Paspalum dilatatum, biotype, pentaploid, hybridization.

\section{INTRODUÇÃO}

O gênero Paspalum tem grande importância no Cone Sul da América do Sul, por englobar gramíneas nativas de bom valor forrageiro e com potencial para melhoramento genético, visando ao estabelecimento de pastagens cultivadas. É caracterizado pela diversidade genética e complexidade, principalmente no que se refere às relações entre os grupos taxonomicamente informais, nos quais o gênero é tradicionalmente dividido (CHASE, 1929; BARRETO, 1974). O grupo Dilatata reúne quatro espécies, cujos genomas básicos (I e J) encontram-se amplamente distribuídos pelo gênero (BURSON \& QUARIN, 1982; VALLS, 2000).Paspalum dilatatum destaca-se, no grupo, como a espécie com maior variabilidade.

Paspalum dilatatum "Comum" corresponde ao tipo nomenclatural da espécie e, por muitos anos, foi considerado o único biótipo de $\boldsymbol{P}$. dilatatum (BURSON et al., 1991). Hoje, estão descritos mais seis biótipos, cada um com combinações peculiares de atributos morfológicos, citológicos e reprodutivos (VALLS \& POZZOBON, 1987). Paspalum dilatatum "Comum" ainda é o de maior interesse, em função de sua distribuição ampla e sua capacidade de produção de forragem de boa qualidade.

Uma vez que a utilidade do germoplasma é diretamente proporcional à qualidade do conhecimento científico a seu respeito (VALLS, 1987), a caracterização biológica de seis acessos discrepantes, pertencentes ao grupo Dilatata, finalidade deste trabalho, foi direcionada ao melhor entendimento da variabilidade existente no grupo e de seus processos de evolução.

\section{MATERIAL E MÉTODOS}

O trabalho consistiu na análise morfológica e citogenética de seis acessos de germoplasma e de representantes de seus prováveis genitores, todos pertencentes ao grupo Dilatata, oriundos de coletas realizadas no Rio Grande do Sul e mantidos em cultivo em vasos, em telado, na Embrapa Recursos Genéticos e Biotecnologia, Brasília, DF.

Para a análise morfológica, foram utilizados descritores de estruturas da inflorescência, incluindo caracteres de importância taxonômica para o grupo. O material foi observado sob microscópio estereoscópio e as medidas de estruturas menores foram obtidas com auxílio de escala milimetrada.

A determinação do número cromossômico se deu a partir da análise mitótica, em, no mínimo, 15 células meristemáticas de ápices radiculares por planta, através de metodologia convencional para o gênero (POZZOBON \& VALLS, 1997).

\section{RESULTADOS E DISCUSSÃO}

A análise mitótica dos seis acessos morfologicamente discrepantes, obtidos em coletas realizadas em anos recentes, levou à detecção de dois novos citotipos pentaplóides, com origem muito provavelmente distinta do biótipo "Comum" de $\boldsymbol{P}$. dilatatum.

Até o momento, $\boldsymbol{P}$. dilatatum biótipo "Comum" era o único pentaplóide descrito para a espécie, constituindo-se no principal alvo de vários programas de melhoramento (BURSON et al., 1973). Presume-se que este pentaplóide tenha-se originado de um cruzamento natural entre o biótipo tetraplóide sexual "Flavescens" (P. dilatatum subsp. flavescens Roseng., Arr. \& Izag.) e o hexaplóide apomítico "Uruguaio" (BURSON, 1983). A distribuição geográfica desses biótipos 4x e 6x sugere que tal evento tenha ocorrido na área hoje correspondente ao Uruguai. Evidências relacionadas ao pareamento meiótico de híbridos obtidos, à distribuição geográfica e a características fenotípicas intermediárias são alguns dos aspectos que fortalecem a hipótese de hibridação acima (BURSON, 1983, 1991; CAPONIO \& QUARIN, 1990). Além disso, estudos com cruzamentos confirmaram que alguns dos cromossomos dos genomas desconhecidos dos biótipos "Comum" e "Uruguaio", designados como X, são similares (BURSON, 1991).

O primeiro dos biótipos pentaplóides discrepantes (acessos V14285 e V14289, do Litoral Atlântico, e V14860, da Depressão Central, todos no Rio Grande do Sul), associa-se geográfica e morfologicamente ao hexaplóide $\boldsymbol{P}$. dilatatum "Torres". Com base em caracteres morfológicos, estes acessos foram originalmente enquadrados no biótipo "Torres", por ocasião de sua coleta, tamanha a similaridade. Porém, a análise citogenética indicou que se tratavam de pentaplóides, nível ainda não relatado para $\boldsymbol{P}$. dilatatum "Torres". A análise morfológica mais aprofundada e a posterior comparação com material de outras coletas, evidenciou que dois destes acessos - V14285, V14289 - mostram diferenças sutis quanto à 
estrutura da inflorescência, como o comprimento do eixo, ligeiramente maior, e maior número de ramos que o observado em acessos típicos do biótipo "Torres" (Tabela 1). Condições ambientais são capazes de ocasionar variações nos caracteres vegetativos e reprodutivos. Além disto, certo grau de variabilidade entre acessos é natural e não implica, necessariamente, em sua discriminação. No entanto, a análise mitótica confirmou tais variações também nos aspectos citológicos, estendendo a discrepância ao nível de ploidia (Figura 1).

Infere-se, portanto, a possibilidade de sua origem por hibridação entre o biótipo hexaplóide $\boldsymbol{P}$. dilatatum "Torres" e algum tetraplóide sexual próximo. Paspalum dilatatum "Torres" é a forma hexaplóide mais freqüente na mesma área, apresentando inflorescências piramidais, com ramos curtos e numerosos (BURSON et al., 1991). Paspalum urvillei é a espécie do grupo Dilatata com maior área de ocorrência no Brasil. Suas inflorescências são extremamente ricas, com ramos numerosos, de comprimento decrescente para o ápice (BURSON, 1979). Considera-se que esta espécie, boa doadora e receptora de pólen (CAPONIO \& QUARIN, 1990), esteja envolvida na origem deste novo pentaplóide. Além disto, $\boldsymbol{P}$. urvillei é a única espécie sexual tetraplóide do grupo Dilatata simpátrica na área de coleta dos referidos acessos pentaplóides. Outra característica da inflorescência, a disposição dos ramos sobre o eixo, também se comporta de forma intermediária entre os prováveis genitores.

A grande semelhança morfológica do acesso V14860 com seu possível genitor $\boldsymbol{P}$. dilatatum "Torres" (Tabela 1) enfatiza a necessidade de contagem cromossômica de cada acesso de germoplasma já obtido ou a ser resgatado deste biótipo. A partir dos dados do presente trabalho, torna-se óbvio que a simples observação da morfologia não mais permite a determinação segura do biótipo "Torres", associada, desde sua primeira citação, à presença de 60 cromossomos (FERNANDES et al., 1968; BURSON, 1991).

O segundo novo pentaplóide (V14253, de Uruguaiana, na região fisiográfica da Campanha do Rio Grande do Sul) foi originalmente identificado, a campo, como pertencente ao biótipo "Virasoro", porém com ressalva quanto ao comprimento da espigueta, muito menor. A análise citogenética mostrou 50 cromossomos. Este acesso deve resultar da hibridação entre plantas dos biótipos "Virasoro" - 4x, sexual (CAPONIO \& QUARIN, 1990; HICKENBICK et al., 1992) e "Uruguaiana" - 6x, apomítico (FERNANDES et al., 1968; BURSON, 1991). Dentre vários caracteres nitidamente intermediários, o aspecto mais distintivo é a presença de sete ou mais nervuras no lema estéril, característica típica do biótipo "Virasoro", mas em espiguetas muito menores, que reportam ao biótipo "Uruguaiana", ocorrente a poucos metros de distância do novo pentaplóide, no sítio original de coleta do acesso V14253 (Tabela2).

Embora o biótipo "Virasoro" ainda não tenha sido coletado em simpatria com $\boldsymbol{P}$. dilatatum "Uruguaiana", este tetraplóide é o mais provável genitor sexual do híbrido V14253, já que o maior número de nervuras no lema, observadas no acesso em questão, é peculiar a este biótipo (Figura 2). Além disto, novas coletas documentaram a ocorrência de $\boldsymbol{P}$. dilatatum "Virasoro" no mesmo município de Uruguaiana.

No munícipio de Alegrete, onde a presença do biótipo "Virasoro" é amplamente documentada em herbário, encontraram-se duas novas plantas que compartilham os mesmos aspectos morfológicos (Tabela 2) do acesso V14253 (acessos V14930 e V14949). Sua análise mitótica produziu resultados similares, implicando em expansão da área de ocorrência conhecida deste novo biótipo pentaplóide. As duas novas plantas foram coletadas em densa

Tabela 1 - Valores médios de caracteres morfológicos relacionados aos híbridos V14285, V14289 e V14860 e seus prováveis genitores do grupo Dilatata.

\begin{tabular}{|c|c|c|c|}
\hline \multirow[b]{2}{*}{ Espécie/Biótipo ${ }^{1,2}$} & \multirow[b]{2}{*}{$2 n$} & \multicolumn{2}{|c|}{ Características morfológicas } \\
\hline & & Comprimento do eixo $(\mathrm{mm})$ & Número de ramos \\
\hline P. urvillei (Média de 12 acessos ) & 40 & 165,0 & 13,0 \\
\hline \multicolumn{4}{|l|}{ P. dilatatum "Torres" х $\boldsymbol{P}$. urvillei (?) } \\
\hline VTsZi 14285 & 50 & 167,0 & 14,0 \\
\hline VTsZi 14289 & 50 & 115,0 & 9,0 \\
\hline VTs 14860 & 50 & 99,0 & 7,4 \\
\hline P. dilatatum "Torres" (Média de 5 acessos) & 60 & 94,9 & 8,0 \\
\hline
\end{tabular}

${ }^{1}$ Apenas acessos que tiveram o número cromossômico determinado foram incluídos na análise morfológica.

${ }^{2}$ Coletores: Ts: T. Souza-Chies; V: J.F.M. Valls; Zi: F. Zílio.

Ciência Rural, v.35, n.1, jan-fev, 2005. 


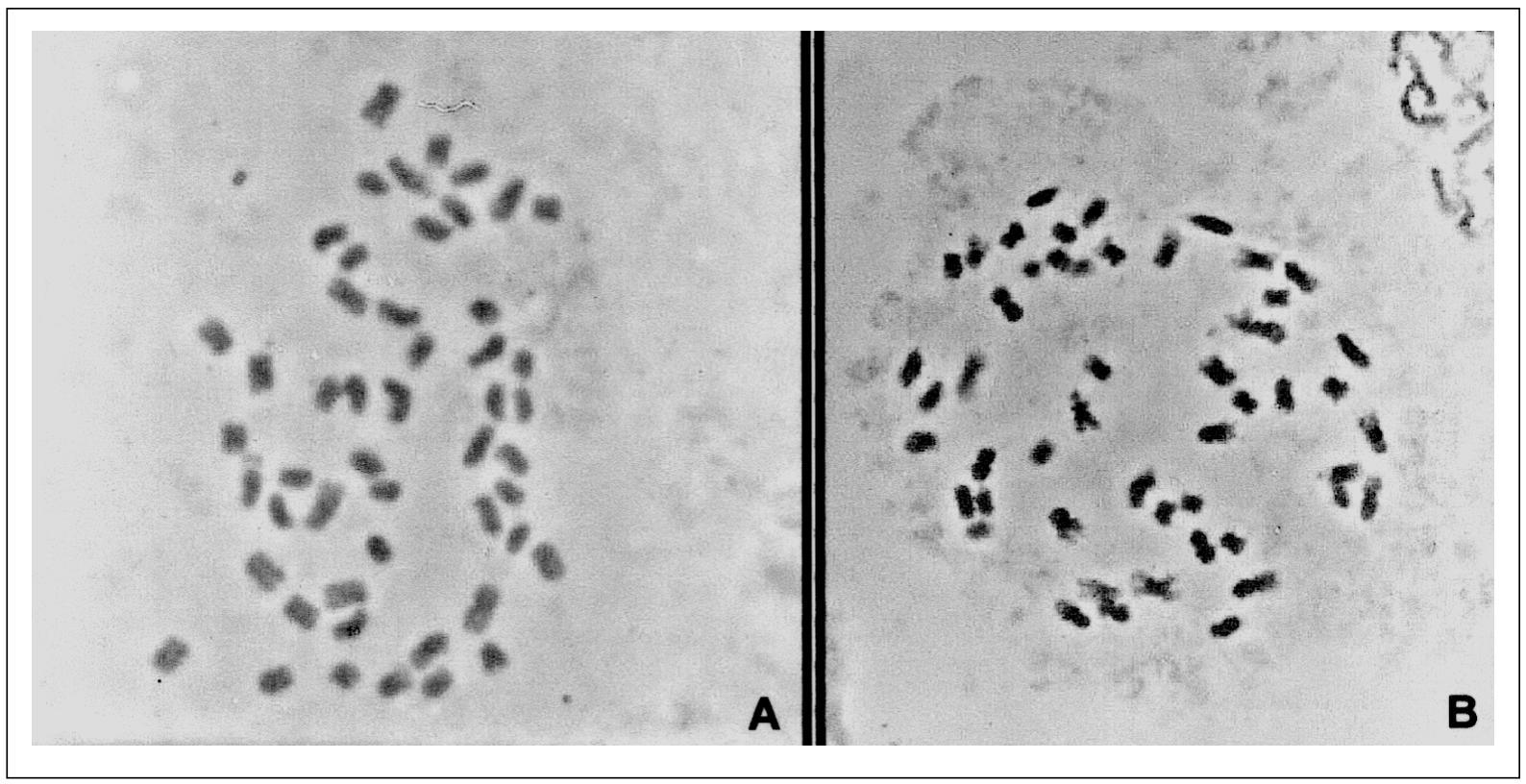

Figura 1 - Células em metáfase mitótica, evidenciando 2n=50 cromossomos. A). P. dilatatum "Torres" х $\boldsymbol{P}$. urvillei (V14289). B). $\boldsymbol{P}$. dilatatum "Virasoro" х $\boldsymbol{P}$. dilatatum "Uruguaiana" (V14253)

associação com touceiras de $\boldsymbol{P}$. urvillei, dando a impressão inicial, por suas anteras roxas e inflorescências ricas, que poderiam tratar-se de híbridos entre $\boldsymbol{P}$. dilatatum "Uruguaiana" e $\boldsymbol{P}$. urvillei, o que, no entanto, é descartado pela morfologia da espigueta observada com pequeno aumento.

$\mathrm{O}$ valor agronômico da forragem e o potencial de persistência destes novos pentaplóides, na natureza e em condições de cultivo, ainda precisam ser verificados, mas, sem dúvida, o encontro destes pentaplóides adicionais tem importância para o entendimento da evolução do grupo, aumentando ainda mais a variabilidade intra e interespecífica disponível para pesquisa do gênero.

\section{CONCLUSÃO}

A descrição destes seis novos acessos do grupo Dilatata e sua classificação em dois novos biótipos pentaplóides sugere uma origem semelhante dos três biótipos pentaplóides ("Comum", "Virasoro" x "Uruguaiana" e "Torres" x $\boldsymbol{P}$. urvillei), todos provavelmente oriundos de cruzamentos entre tetraplóides sexuais e hexaplóides apomitícos do grupo Dilatata, mas a partir de três combinações distintas. No entanto, não havia registro anterior da ocorrência de evento desta natureza no Brasil. Pode-se especular que haja mais combinações híbridas de ocorrência natural, ainda não disponíveis em coleções, por falta

Tabela 2 - Valores médios de caracteres morfológicos relacionados aos híbridos V14253, V14930 e V14949 e seus prováveis genitores do grupo Dilatata de Paspalum.

\begin{tabular}{|c|c|c|c|}
\hline \multirow{2}{*}{ Espécie/Biótipo ${ }^{1,2}$} & \multirow{2}{*}{$2 n$} & \multicolumn{2}{|c|}{ Características morfológicas } \\
\hline & & Comprimento da espigueta (mm) & Número de nervuras \\
\hline P. dilatatum "Virasoro" (Média de 15 acessos) & 40 & 4,4 & 10,2 \\
\hline \multicolumn{4}{|l|}{ P. dilatatum "Virasoro" x "Uruguaiana" (?) } \\
\hline VTsDp 14253 & 50 & 3,8 & 9,0 \\
\hline VLiGuSo 14930 & 50 & 3,5 & 7,4 \\
\hline VLiGuSo 14949 & 50 & 3,6 & 7,7 \\
\hline P. dilatatum "Uruguaiana" (Média de 3 acessos) & 60 & 3,4 & 4,6 \\
\hline
\end{tabular}

${ }^{1}$ Apenas acessos que tiveram o número cromossômico determinado foram incluídos na análise morfológica.

${ }^{2}$ Coletores: Dp: D. Palmieri; Gu: A. Guglieri; Li: L. Essi; So: S. Hefler; Ts: T. Souza-Chies; V: J.F.M.Valls.

Ciência Rural, v.35, n.1, jan-fev, 2005. 


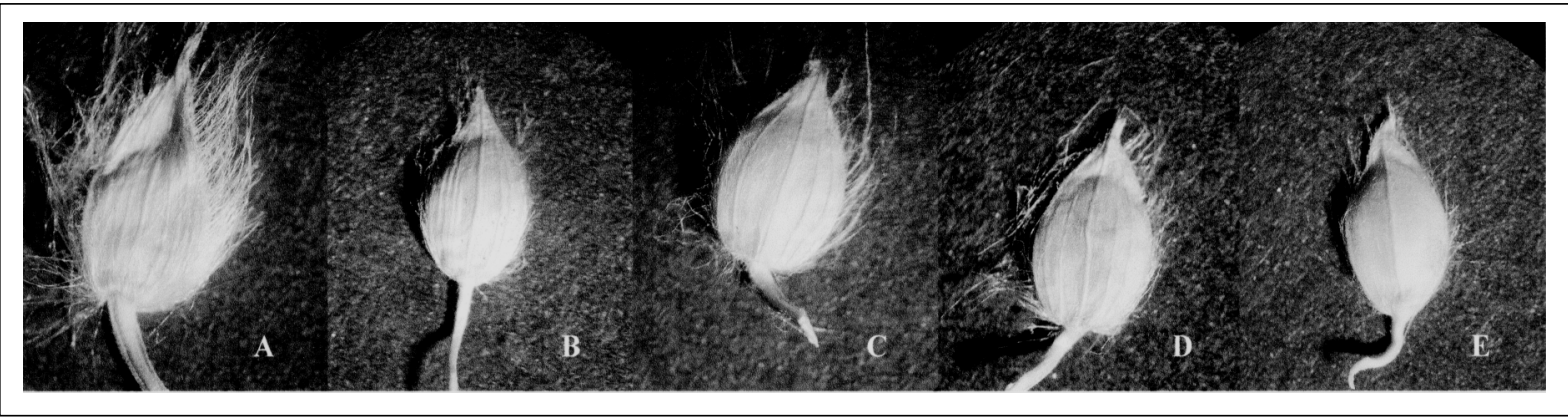

Figura 2 - Aspectos morfológicos das espiguetas de Paspalum dilatatum "Virasoro" (A), P. dilatatum "Uruguaiana" (E) e seus híbridos pentaplóides: V14253 (B); V14930 (C); V14949 (D). Todas as espiguetas seguem a mesma escala.

de coletas direcionadas, ou já disponíveis, mas não detectadas, por falta de caracterização adequada. Além disso, o encontro dos novos acessos pentaplóides em sítios distantes e a postulação de sua origem por cruzamentos naturais entre biótipos tetraplóides sexuais e hexaplóides apomíticos sugerem que a produção de gametas masculinos balanceadamente reduzidos, em indivíduos que se reproduzem por apomixia, não seja um evento raro.Coletas intensas e programas persistentes de caracterização, mesmo de espécies com germoplasma abundante, são ações necessárias, já que a busca contínua pode revelar boas características reprodutivas ou forrageiras em material ainda não utilizado, ou esclarecer aspectos importantes acerca da evolução do gênero Paspalum, podendo trazer à luz novas formas biológicas com potencial de aproveitamento.

\section{AGRADECIMENTOS}

Este trabalho contou com recursos aportados pela Embrapa, Universidade Federal do Rio Grande do Sul (UFRGS), Conselho Nacional de Desenvolvimento Científico e Tecnológico (CNPq) e Fundação de Amparo à Pesquisa do Estado do Rio Grande do Sul (FAPERGS), através de projetos liderados pelo segundo autor e pela $\operatorname{Prof}^{\underline{a}} \operatorname{Dr}^{\underline{a}}$ Tatiana T. de Souza-Chies (Departamentos de Genética e Botânica /UFRGS).

\section{REFERÊNCIAS BIBLIOGRÁFICAS}

BARRETO, I.L. O gênero Paspalum L. (Gramineae) no Rio Grande do Sul. 1974. 258f. Tese (Livre Docência) Universidade Federal do Rio Grande do Sul.

BURSON, B.L. Cytogenetics of Paspalum urvillei $\mathrm{x} \boldsymbol{P}$. intermedium and $P$. dilatatum $\times$ P. paniculatum hybrids. Crop Science, Madison, v.19, p.534-538, 1979.

BURSON, B.L. Phylogenetic investigations of Paspalum dilatatum and related species. In: INTERNATIONAL GRASSLAND CONGRESS, 14., 1983, Lexington, KY. Proceedings... Boulder : Westview, 1983. p.170-173.
BURSON, B.L. Genome relationships between tetraploid and hexaploid biotypes of Dallisgrass, Paspalum dilatatum. Botanical Gazette. Chicago, v.152, p.219-223, 1991.

BURSON, B.L.; QUARIN, C.L. Cytology of Paspalum virgatum and its relationships with $\boldsymbol{P}$. intermedium and $\boldsymbol{P}$. juergensii. Canadian Journal of Genetics and Cytology Ottawa, v.24, p.219-226, 1982.

BURSON, B.L. et al. Genome relations between tetraploid Paspalum dilatatum and four diploid Paspalum species. Crop Science, Madison, v.13, p.739-743, 1973.

BURSON, B.L. et al. Cytology, reproductive behavior and forage potential of hexaploid Dallisgrass biotypes. Crop Science, Madison, v.31, p.636-641, 1991.

CAPONIO, I.; QUARIN, C.L. Intra and interespecific hybridization between Dallisgrass and Vaseygrass. Crop Science, Madison, v.30, p.362-364, 1990.

CHASE, A. The North American species of Paspalum. Contributions from the United States National Herbarium, Washington, v.28, p.1-310. I-XVII, 1929.

FERNANDES, M.I.B.M. et al. Cytogenetics, ecologic and morphologic studies in Brazilian forms of Paspalum dilatatum. Canadian Journal of Genetics and Cytology, Ottawa, v.10, p.131-138, 1968.

HICKENBICK, M.C.M. et al. Mode of reproduction and seed production in Paspalum dilatatum Poir. Virasoro biotype Dilatata Group (Gramineae). Revista Brasileira de Genética, Ribeirão Preto, v.15, p.85-102, 1992.

POZZOBON, M.T.; VALLS, J.F.M. Chromosome numbers in germplasm accessions of Paspalum notatum (Gramineae). Brazilian Journal of Genetics, v.20, p.2934, 1997.

VALLS, J.F.M. Recursos genéticos de espécies de Paspalum no Brasil. In: ENCONTRO INTERNACIONAL SOBRE MELHORAMENTO GENÉTICO DE PASPALUM, 1987, Nova Odessa, SP. Anais... Nova Odessa : Instituto de Zootecnia, 1987. 89p. p.3-13.

VALLS, J.F.M. Impacto do conhecimento citogenético na taxonomia de Paspalum e Axonopus (Gramineae). In: 
CAVAlCANTI, T.B.; WAlTer, B.M.T. (Org). Tópicos atuais em botânica. Brasília : SBB/Embrapa Recursos e Biotecnologia, 2000. p.57-60.

VALLS, J.F.M.; POZZOBON, M.T. Variação apresentada pelos principais grupos taxonômicos de Paspalum com interesse forrageiro no Brasil. In: ENCONTRO INTERNACIONAL SOBRE MELHORAMENTO GENÉTICO DE PASPALUM, 1987, Nova Odessa, SP. Anais... Nova Odessa : Instituto de Zootecnia, 1987. 89p. p.15-21. 